\title{
EVALUACIÓN DE LA CALIDAD DE VIDA RELACIONADA AL CLIMATERIO Y MENOPAUSIA.
}

\section{EVALUATION OF THE QUALITY OF LIFE RELATED TO CLIMACTERIC AND MENOPAUSE.}

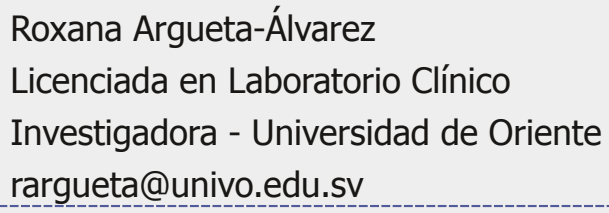

\section{Resumen}

El climaterio es un periodo de transición que pone término a la capacidad reproductiva de la mujer. Inicia antes de que se produzca el cese del periodo menstrual; mientras que la menopausia se caracteriza por ausencia total, provocando alteraciones somáticas, psicológicas y genitourinarias. Objetivo: Determinar la calidad de vida de las mujeres en la etapa del climaterio y la menopausia. Metodología: Estudio cuantitativo de alcance descriptivo, en mujeres de la zona oriental, con criterio de inclusión de edades entre 45 y 55 años. El instrumento utilizado fue la Escala de Puntuación Menopáusica (Menopause Rating Scale, MRS), incluyendo preguntas relacionadas a la salud en general. Principios éticos: previo consentimiento informado de cada una de las participantes, respetando el derecho de confidencialidad. Resultados: Participaron 123 mujeres, los síntomas más frecuentes, con escala de valor desde leve a intolerable, son las molestias musculares y articulares ( $86 \%$ ) a ellas le sigue el cansancio físico y mental (82\%) y la irritabilidad (76\%). Según los dominios que componen la escala MRS, se presentan con mayor frecuencia los de naturaleza psicológica, paralelamente relacionados al somato vegetativo. Conclusiones / Recomendaciones: el diagnóstico de patologías como hipertensión y diabetes en las pacientes influye en la severidad de la sintomatología, repercutiendo directamente en la calidad de vida de las mismas. Es importante considerar la vulnerabilidad de la familia dentro de los programas de atención a la mujer en este proceso, contribuyendo a la asimilación y mejora de la calidad de vida de la mujer.

Palabras clave: Calidad de vida, climaterio, menopausia.

\section{Abstract}

This work aims to determine the quality of life of women in the climacteric and menopause. Climacteric is a transition period 
that ends the women's reproductive capacity. This process begins before the cessation of period, while menopause is characterized by a total absence, causing somatic, psychological and genitourinary disorders. The study methodology was quantitative with descriptive scope in 123 women in the eastern zone, with age considerations between 45 and 55 years old. The instrument used was the Menopause Rating Scale; Scale, (MRS), which included questions related to general health. The ethical principles of prior informed consent were used for each of the participants, respecting the right to confidentiality. The results obtained highlight that, the most frequent, with a scale of value from mild to intolerable, are the muscular and the first is the lack of physical and mental fatigue (82\%), followed by irritability (76\%).

According to the domains that make up the MRS scale, are most often those of a psychological, parallel to the vegetative somato. The diagnosis of pathologies such as hypertension and diabetes in patients influence the severity of the symptomatology, directly impacting on their quality of life.

Keywords: Women and health, quality of life, climacteric, menopause.

\section{Introducción}

La mujer, a lo largo de la vida, experimenta cambios significativos, que en ocasiones condicionan de manera considerable su bienestar.

A pesar de haber sido ignorada por mucho tiempo, y hasta subestimada tanto por las propias mujeres como también por muchos profesionales a través de diferentes épocas, la etapa del climaterio y la menopausia ha sido motivo de menciones históricas, literarias, culturales y de mayor interés aún para los investigadores. Son muy antiguas las citas que hay sobre los síntomas y las molestias propias de la mujer en esta etapa de la vida, y que siempre alertaban y relacionaban de manera constante acerca del final de la vida reproductiva.

La menopausia se convierte en una de las etapas que conjuga una serie de eventos que repercuten en su bienestar. Si bien se trata de una etapa de la vida femenina, y por consiguiente de un proceso fisiológico normal, no deben pasarse por alto los eventos que rodean este periodo, en el cual se da el aparecimiento de múltiples manifestaciones clínicas que impactan de manera contundente la calidad de vida de la mujer (Sordia Hernández , 2009, pág. 7).

Todos estos cambios la mujer los percibe e influyen sobre su imagen, autoestima, vida social, laboral y familiar, entre otras esferas. En este periodo, se puede alterar la habilidad y disposición de muchas actividades debido las manifestaciones vasomotoras que lo 
acompañan, caracterizadas por bochornos, rubor y sudoración a predominio nocturno, que pueden llegar a ser muy molestas. Asimismo se acentúa el insomnio que acompaña a las alteraciones neuro-psíquicas, producien-dovirritabilidad, cambios de carácter, pérdida de la concentración o memoria, y disminución o ausencia del deseo sexual (Urdaneta, Cepeda de V., Guerra , Baabel , \& Conteras, 2010).

Para mantener su equilibrio físico y psicológico ha de estar preparada emocional y racionalmente. Es una situación nueva que tiene que afrontar, no exenta de interrogantes e inseguridades en las que en muchas ocasiones no se busca solución debido a la poca información de importancia, tanto de parte de los miembros de su familia como de la propia mujer, considerando el proceso como un periodo de transición que es inevitable.

El Ministerio de Salud en la Guía de Atención Clínica del Climaterio y la menopausia menciona que, según la encuesta Nacional de Salud Familiar (FESAL 2002-2003), la esperanza de vida para las mujeres, es de 73 años. En El Salvador se estima una población de 6.6 millones de habitantes, donde el $50.9 \%$ son mujeres, y de ellas el $11 \%$ son mayores de 49 años; es decir que alrededor de 300,000 salvadoreñas enfrentan los problemas propios de su edad, incluyendo los relacionados al climaterio y menopausia. (Ministerio de Salud Publica y Asistencia
Social, 2008). La edad promedio en que se presenta la menopausia es de 50 años, aunque esto varía de una mujer a otra; esto implica que un gran porcentaje de mujeres salvadoreñas vivirán un promedio de 23 o más años después de su menopausia. Lo que llevara a la presencia más frecuente de sintomatología propia de la peri y postmenopausia, así como el riesgo de desarrollar complicaciones que deterioren su estado de salud.

En la búsqueda de una solución es frecuente que las mujeres consulten al médico sobre la menopausia, y en general, los médicos se dediquen a los problemas biológicos, salvo que estas manifiesten otro problema agregado. En la mayoría de las veces se realiza una evaluación puramente sintomática y rápida al problema, dando como respuesta la medicación a sus quejas; pero no se considera el abordaje del área psicológica y social que afecta su autoestima y calidad de vida.

Aunque la menopausia no es una condición patológica que tenga que ser tratada, es necesario la ayuda en ciertos síntomas, como los calores súbitos, la falta de apetito, el insomnio y los cambios menstruales, agregados aquí todos aquellos síntomas como palpitaciones, náuseas, dolor de cabeza, depresión y resequedad vaginal entre otros. Optar por una terapia de reemplazo durante este proceso es de suma importancia para 
mejorar la calidad de vida de las mujeres.

\section{Metodología}

El enfoque metodológico de la investigación es cuantitativo de alcance descriptivo. Roberto Sampieri denomina a la investigación cuantitativa como 'la investigación que utiliza la recolección de datos para probar hipótesis con base en la medición numérica y el análisis estadístico, con el fin de establecer pautas de comportamiento y probar teorías' (Hernández Sampieri, 20I4, pág. 4). La muestra estuvo constituida por 123 mujeres de los diferentes municipios de la zona oriental con un criterio de inclusión que debían estar entre las edades de 45 a 55 años y sintomatología específica relacionada al climaterio. El instrumento utilizado fue la escala MRS, la cual está compuesta por once síntomas, agrupados en tres dominios: somático-vegetativo, psicológico y urogenital. El dominio somático vegetativo incluye bochornos, molestias cardíacas, dificultades del sueño, molestias musculares y articulares. En los síntomas psicológicos, se agrupan depresión, irritabilidad, ansiedad, agotamiento físico y mental. Por último, los síntomas urogenitales, incluyen los problemas sexuales, de vejiga y sequedad vaginal. Cada síntoma es autoevaluado por la mujer con un puntaje de 0 a 4 según el grado de intensidad: $0=\sin$ molestia; I $=$ molestia leve; 2 = molestia moderada; $3=$ molestia severa; $4=$ molestia intolerable.
Para dar cumplimiento a los principios éticos internacionales en investigación, se consideró la categoría de la investigación la cual es sin riesgo: estudio que emplea técnicas y métodos de investigación documental retrospectivos y aquellos en los que no se realiza ninguna intervención $\circ$ modificación intencionada en las variables fisiológicas, psicológicas y sociales de los individuos que participan en el estudio (Ministerio de Salud, Instituto Nacional de Salud, 2017, pág. 15). Con previo conocimiento informado de las participantes.

Los datos obtenidos serán de interés y carácter académico, procesándose en tablas de distribución de frecuencias y representaciones gráficas de los valores obtenidos en cada ítem evaluado a través del software estadístico Excel 2013.

\section{Análisis e interpretación de resultados}

A continuación, se presentan los resultados de 123 mujeres, que participaron en este estudio, con criterio de inclusión de edad que oscila entre 45 a 55 años; de los cuatro departamentos que conforman la zona oriental.

\section{Datos sociodemográficos}

La población participante son mujeres de los cuatro departamentos que conforman la zona oriental: San Miguel, Morazán, La Unión y Usulután; reflejando mayor participación en 
este estudio de mujeres del departamento de San Miguel, donde el $77 \%$ oscilan entre las edades de 45 a 50 años el resto se encuentra entre $5 \mathrm{I}$ a 55 años.

Figura 1. Distribución de la población con base al departamento de origen y edad

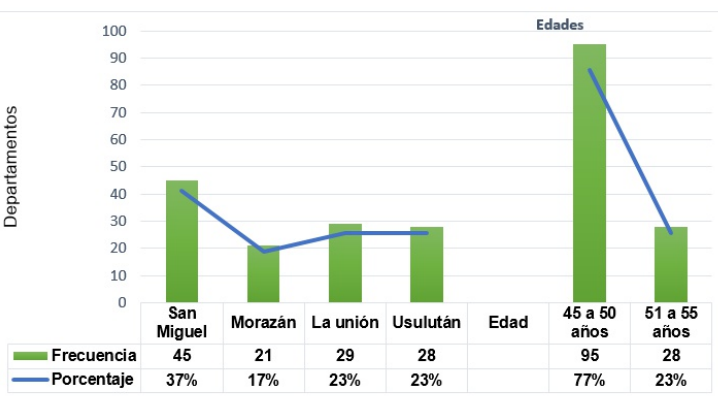

Fuente: Elaboración propia

\section{Características relacionadas a la salud en general}

Se realizan algunas consultas con la finalidad de explorar generalidades sobre la salud de las participantes relacionadas al proceso del climaterio y la menopausia que infieren en la calidad de vida de las mujeres en esta etapa.

\section{Figura 2. Fecha de ultima menstruación}

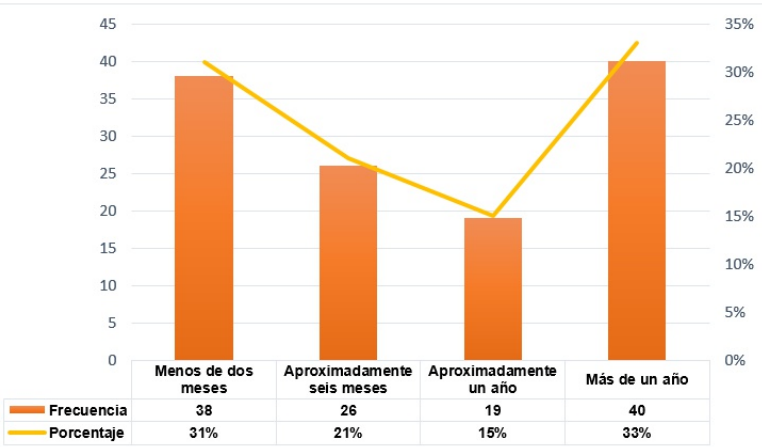

Fuente: Elaboración propia
Figura 3: Participantes que se encuentra bajo algún tratamiento de planificación

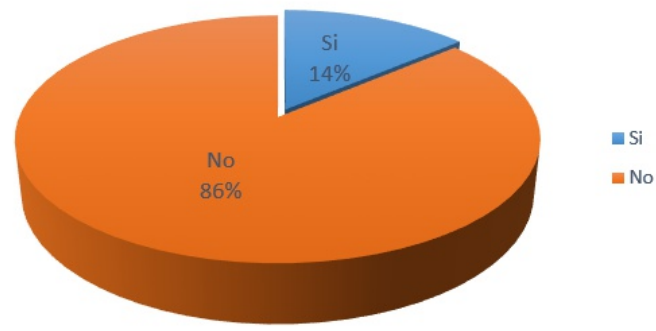

Fuente: Elaboración propia

Es importante considerar en este estudio la fecha de última menstruación, así como el tratamiento bajo algún método de planificación. El $69 \%$ de las mujeres encuestadas tienen más de seis meses de cese del periodo menstrual. Este punto es significativo porque define el inicio del climaterio o diagnóstico de menopausia. Para este caso, es de interés destacar que han iniciado el proceso del climaterio el $42 \%$ de ellas, el resto ya alcanzó la menopausia. Es necesario considerar que el $86 \%$ no está planificando, ya que esto sería aún determinante en este proceso, debido a que los métodos de planificación pueden causar trastornos menstruales que podrían confundirse con el climaterio.

Figura 4. Patologías más frecuentes en etapa del climaterio y menopausia

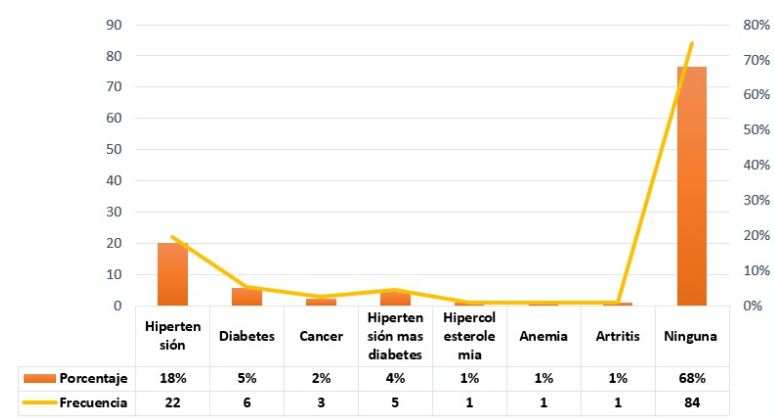

Fuente: Elaboración propia 
La menopausia es asociada con diversos padecimientos $y$ enfermedades, debido a que es en esta etapa cuando la producción de hormonas, como estrógenos y progesterona, disminuye considerablemente, afectando la función de diferentes glándulas que provocan cambios en el organismo. La patología más frecuente en esta población es la hipertensión seguida de la diabetes, o ambas. En algunos casos; influyendo directamente en la calidad de vida de la mujer que no solo le corresponde lidiar con los síntomas propios de este proceso sino también con patologías asociadas a la misma etapa.

\section{Frecuencia de la sintomatología} según escala MRS

Se detalla a continuación la frecuencia de la sintomatología referida por las participantes según la escala de evaluación MRS; utilizada para evaluar la calidad de vida de las mujeres en etapa de la menopausia.

Tabla 1: Frecuencia de los síntomas referidos según valoración de escala MRS

\begin{tabular}{|c|c|c|c|c|c|c|}
\hline \multirow[t]{2}{*}{ Sintomas } & \multirow{2}{*}{$\begin{array}{c}\text { Escala de } \\
\text { Puntuación }\end{array}$} & 0 & 1 & 2 & 3 & 4 \\
\hline & & Sin molestia & Leve & Moderado & Severo & Intolerable \\
\hline \multirow[t]{2}{*}{ Bochornos } & Frecuencia & 33 & 31 & 36 & 12 & 11 \\
\hline & Porcentaje & $27 \%$ & $25 \%$ & $29 \%$ & $10 \%$ & $9 \%$ \\
\hline \multirow{2}{*}{$\begin{array}{l}\text { Molestias al } \\
\text { corazón }\end{array}$} & Frecuencia & 40 & 25 & 34 & 13 & 11 \\
\hline & Porcentaje & $32 \%$ & $20 \%$ & $28 \%$ & $11 \%$ & $9 \%$ \\
\hline \multirow{2}{*}{$\begin{array}{l}\text { Molestias } \\
\text { musculares } \\
\text { y articulares }\end{array}$} & Frecuencia & 17 & 32 & 36 & 17 & 21 \\
\hline & Porcentaje & $14 \%$ & $26 \%$ & $29 \%$ & $14 \%$ & $17 \%$ \\
\hline \multirow{2}{*}{$\begin{array}{l}\text { Dificultades } \\
\text { en el sueño }\end{array}$} & Frecuencia & 40 & 30 & 15 & 17 & 21 \\
\hline & Porcentaje & $33 \%$ & $24 \%$ & $12 \%$ & $14 \%$ & $17 \%$ \\
\hline \multirow{2}{*}{$\begin{array}{l}\text { Estado de } \\
\text { ánimo } \\
\text { depresivo }\end{array}$} & Frecuencia & 42 & 26 & 19 & 20 & 16 \\
\hline & Porcentaje & $34 \%$ & $21 \%$ & $16 \%$ & $16 \%$ & $13 \%$ \\
\hline \multirow[t]{2}{*}{ Irritabilidad } & Frecuencia & 29 & 30 & 30 & 19 & 15 \\
\hline & Porcentaje & $24 \%$ & $24 \%$ & $24 \%$ & $16 \%$ & $12 \%$ \\
\hline \multirow[t]{2}{*}{ Ansiedad } & Frecuencia & 35 & 36 & 26 & 15 & 11 \\
\hline & Porcentaje & $29 \%$ & $29 \%$ & $21 \%$ & $12 \%$ & $9 \%$ \\
\hline \multirow{2}{*}{$\begin{array}{l}\text { Cansancio } \\
\text { físico y } \\
\text { mental }\end{array}$} & Frecuencia & 22 & 38 & 30 & 19 & 14 \\
\hline & Porcentaje & $18 \%$ & $31 \%$ & $24 \%$ & $16 \%$ & $11 \%$ \\
\hline \multirow{2}{*}{$\begin{array}{l}\text { Problemas } \\
\text { sexuales }\end{array}$} & Frecuencia & 50 & 33 & 15 & 11 & 14 \\
\hline & Porcentaje & $41 \%$ & $27 \%$ & $12 \%$ & $9 \%$ & $11 \%$ \\
\hline \multirow{2}{*}{$\begin{array}{l}\text { Problemas } \\
\text { de vejiga }\end{array}$} & Frecuencia & 69 & 15 & 14 & 13 & 12 \\
\hline & Porcentaje & $56 \%$ & $12 \%$ & $11 \%$ & $11 \%$ & $10 \%$ \\
\hline \multirow{2}{*}{$\begin{array}{l}\text { Sequedad } \\
\text { vaginal }\end{array}$} & Frecuencia & 52 & 27 & 23 & 11 & 10 \\
\hline & Porcentaje & $42 \%$ & $22 \%$ & $19 \%$ & $9 \%$ & $8 \%$ \\
\hline
\end{tabular}


Entre los síntomas más frecuentes con escala seguidos del cansancio físico y mental con un de valor desde las molestias más leves a $82 \%$, y por irritabilidad, en un $76 \%$. intolerables, se destacan las molestias musculares y articulares, con un $86 \%$,

Tabla 2: Calidad de vida por dominio MRS

\begin{tabular}{|l|c|c|c|c|c|c|}
\hline \multirow{2}{*}{$\begin{array}{c}\text { Dominios } \\
\text { MRS }\end{array}$} & Escala de & \multicolumn{1}{|c}{$\mathbf{0}$} & $\mathbf{1}$ & $\mathbf{2}$ & $\mathbf{3}$ & $\mathbf{4}$ \\
\hline & puntuación & Sin molestia & leve & Moderado & Severo & Intolerable \\
\hline \multirow{2}{*}{ Somático } & Frecuencia & 130 & 118 & 121 & 59 & 64 \\
\cline { 3 - 7 } & Porcentaje & $26 \%$ & $24 \%$ & $25 \%$ & $12 \%$ & $13 \%$ \\
\hline Psicológico & Frecuencia & 128 & 130 & 105 & 73 & 56 \\
\hline & Porcentaje & $26 \%$ & $27 \%$ & $21 \%$ & $15 \%$ & $11 \%$ \\
\hline & Frecuencia & 171 & 75 & 52 & 35 & 36 \\
\hline & Porcentaje & $46 \%$ & $20 \%$ & $14 \%$ & $10 \%$ & $10 \%$ \\
\hline
\end{tabular}

Fuente: Elaboración propia

Existe un deterioro en la calidad de vida, desde leve a intolerable, en relación a la frecuencia de las quejas climatéricas, según los dominios de síntomas que componen la escala MRS. Se verificó que los más frecuentes fueron los de naturaleza psicológica, siendo mencionados por un $74 \%$ de encuestadas, paralelamente con los síntomas relacionados con el carácter somato-vegetativo. Las quejas relacionadas a la esfera urogenital se registran en menor porcentaje, pero no por ello son menos importante $54 \%$.

\section{Conclusiones}

A manera de análisis se realizan las siguientes conclusiones a partir de los resultados de esta investigación.

»El síntoma más referido en el dominio somático corresponde a las molestias musculares y articulares; en el dominio psicológico el síntoma más referido es el cansancio físico y mental; y en el dominio urogenital, el síntoma más referido son los problemas sexuales.

"La presencia de patologías como hipertensión y diabetes en las pacientes influye en la severidad de la sintomatología, repercutiendo directamente en la calidad de vida de las mismas.

"La opción de un tratamiento de reemplazo hormonal no es prioritaria en este grupo, considerando lo agudo de la sintomatología que presentan $y$ al porcentaje bajo de mujeres que se encuentran bajo un tratamiento hormonal traduciéndose en una peor calidad de vida para estas mujeres 


\section{Recomendaciones}

„El estudio de la calidad de vida de las mujeres en menopausia se debe extender desde el ámbito poblacional hasta el individual, en los programas de atención primaria. El entendimiento de los síntomas climatéricos y de cómo afectan dicha calidad puede contribuir a mejorar la atención, a descubrir estrategias para el tratamiento de los síntomas y a promover estilos de vida que disminuyan su incidencia y gravedad.

„Es importante considerar la vulnerabilidad de la familia dentro de los programas de atención a la mujer en este proceso, contribuyendo a la asimilación y mejora en la calidad de vida de la mujer.

»Es necesario incluir en una investigación posterior a las pacientes climatéricas que actualmente se encuentren bajo terapia hormonal de reemplazo, $y$ efectuar un seguimiento a mediano y largo plazo para identificar con oportunidad los beneficios inherentes al tratamiento de sustitución.

\section{Bibliografía}

Hernández Sampieri, R. (2014). Metodologia de la Investigacion, Sexta edicion. Mexico: McGrawHill/Interamericana Editores, S.A de C.V. Ministerio de Salud Publica y Asistencia Social. (Febrero de 2008). Centro Virtual de Documentacion Reguladora, Ministerio de Salud. Obtenido de http://asp.salud.gob.sv/regulacion/ pdf/guia/Guia_climaterio_y_menopausia_p1.pdf

Ministerio de Salud, Instituto Nacional de Salud. (2017). Manual de Procedimientos de Investigación en Salud. San Salvador .

Sordia Hernández , L. (Abril de 2009). TRD Tesis
Doctorales en Red. Obtenido de Menopausia: La severidad de su sintomatología y depresión:http:// www.tdx.cat/handle/10803/4654

Urdaneta, J., Cepeda de V., M., Guerra , M., Baabel , N., \& Conteras , A. (2010). Editorial Scielo. Obtenido de: Calidad de vida en mujeres menopausicas con y sin terapia de reemplazo hormonal: http://www.scielo.cl/scielo.php?

script=sci_arttext\&pid=S0717-

75262010000100004 